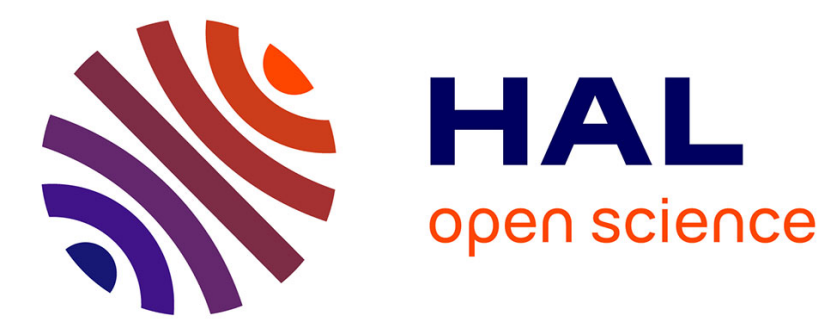

\title{
Moscou : les mutations d'une capitale (1990-2000)
}

Denis Eckert

\section{To cite this version:}

Denis Eckert. Moscou: les mutations d'une capitale (1990-2000). La Revue russe, 2001, pp.29 - 36. 10.3406/russe.2001.2093 . halshs-02566172

\section{HAL Id: halshs-02566172 https://shs.hal.science/halshs-02566172}

Submitted on 6 May 2020

HAL is a multi-disciplinary open access archive for the deposit and dissemination of scientific research documents, whether they are published or not. The documents may come from teaching and research institutions in France or abroad, or from public or private research centers.
L'archive ouverte pluridisciplinaire HAL, est destinée au dépôt et à la diffusion de documents scientifiques de niveau recherche, publiés ou non, émanant des établissements d'enseignement et de recherche français ou étrangers, des laboratoires publics ou privés. 


\section{Moscou : les mutations d'une capitale (1990-2000)}

Denis Eckert

\section{Citer ce document / Cite this document :}

Eckert Denis. Moscou : les mutations d’une capitale (1990-2000). In: Revue Russe n¹9, 2001. La Russie : un autre regard. pp. 29-36;

doi : https://doi.org/10.3406/russe.2001.2093

https://www.persee.fr/doc/russe_1161-0557_2001_num_19_1_2093

Fichier pdf généré le 30/03/2018 


\section{Denis ECKERT}

\section{Moscou : les mutations d'une capitale}

\section{(1990-2000)}

\section{Introduction.}

Moscou a connu des transformations structurelles considérables dans les dix dernières années. La ville occupait une position éminente sur la scène internationale à la fin de la période soviétique : elle cumulait les fonctions de capitale de l'U.R.S.S., puissance nucléaire, de capitale du camp socialiste ; elle était aussi le siège du Comecon et du pacte de Varsovie. Toutes ces fonctions internationales prestigieuses ont disparu avec l'U.R.S.S. ; cela aurait pu affecter de façon très importante le rayonnement de la ville. Or la ville a montré une capacité remarquable à rebondir. Le système économique a changé de base, les paysages urbains se sont profondément modifiés, alors même que le pays dans son ensemble était plongé dans la crise, confronté à de graves difficultés d'adaptation à l'économie de marché. Depuis un ou deux ans, la croissance économique repart, on est dans une configuration un peu plus favorable pour la Russie, du fait du relèvement des cours des matières premières qu'elle exporte. Mais déjà dans les pires années de crise, la ville s'était transformée à une vitesse étonnante, alors même qu'on aurait pu la croire menacée de déclin.

En tant que géographe, j'essaie de comprendre les traits principaux de cette évolution et notamment de saisir en quoi elle affecte les paysages urbains. Quelle est la Moscou qui se dessine aujourd'hui, après dix ans de bouleversements, et qui a œuvré à cette transformation ? Quels sont les pouvoirs économiques et politiques qui ont remodelé cette ville? Après avoir identifié les acteurs de cette transformation, on a aussi envie de faire émerger une autre question sous-jacente : quelle idéologie urbaine est en jeu ? Quel est le projet de ces acteurs, que veulent-ils faire de cette ville ? Moscou a eu naguère la fonction de laboratoire urbain pour l'Union soviétique et elle conserve un peu cette fonction éminente de champ d'expérimentation des nouvelles solutions, des nouvelles architectures. Je souhaite donc présenter une analyse socio-économique et une analyse idéologique des transformations urbaines.

Denis Eckert est chercheur au C.N.R.S. au Centre interdisciplinaire d'études urbaines de l'université de Toulouse - le Mirail. 


\section{La situation socio-économique de la ville.}

Moscou est restée une grande capitale, lieu du pouvoir central dans l'État le plus étendu du monde. Le paysage urbain reste marqué par la présence forte des institutions fédérales. Mais la ville est aussi le lieu du pouvoir économique. C'est là que se trouvent les sièges des très grandes entreprises russes, même celles du secteur énergétique, qui tirent leur prospérité de gisements sibériens pourtant lointains. Ce n'est pas parce que Gazprom, Lukoil, Yukos ont leurs champs d'exploitation loin dans l'Est du pays ou dans le Nord que les dirigeants de ces entreprises ont envisagé de s'implanter ailleurs qu'à Moscou. Les grandes entreprises qui se sont constituées sur les débris des ministères de branches soviétiques ont toutes implanté leurs sièges sociaux au cœur de Moscou. Les acteurs des nouveaux secteurs économiques (banque, services aux entreprises, commerce de gros) ont eu exactement la même logique d'implantation ; des groupes financiers moscovites ont constitué des holdings en rachetant peu à peu un certain nombre de grandes entreprises provinciales, les plus intéressantes, les plus rentables. On est dans un cas de concentration du pouvoir économique qui rappelle d'assez près la concentration exclusive des sièges sociaux des très grandes entreprises françaises à Paris. Ce modèle est bien connu des Français, alors qu'il étonne toujours les Allemands ou les Amériçains qui ont une autre géographie économique.

Les entreprises étrangères ont eu la même analyse : toutes les grandes multinationales qui ont procédé à une implantation sur ce marché, qu'elles soient européennes, américaines ou japonaises ont créé une filiale de droit russe ayant son siège à Moscou. Elles ont par leurs décisions renforcé encore la fonction centrale de la ville dans l'économie nationale. Sur la liste des grandes entreprises présentes à Moscou, on retrouve la fine fleur du capitalisme mondial. C'est à partir de là que les grandes entreprises internationales, sans exception, organisent leur pénétration du marché russe.

Ce choix presque exclusif en faveur de Moscou fait par toute une gamme d'acteurs privés s'explique certainement en grande partie par la concentration de fonctions logistiques : il y avait là dès le départ des aéroports, des infrastructures ferroviaires et de télécommunications, mais également d'importantes surfaces d'entrepôts, de stockage, des centres de dédouanement, un ensemble unique en Russie par sa variété et ses dimensions. On comprend pourquoi Moscou a su capter le meilleur de la transformation structurelle de l'ensemble du pays.

Ce phénomène de concentration a des conséquences évidentes sur l'économie de la ville; même dans les années les plus sombres, Moscou a représenté un îlot de prospérité relative, notamment avec des salaires qui étaient et continuent à être meilleurs qu'ailleurs. Ces salaires sont d'autre part plus souvent versés en liquide - le troc, le versement de salaires en nature sont des procédés beaucoup moins fréquents que dans le reste du pays. La population a donc un pouvoir d'achat très supérieur. Cela se marque de façon frappante, presque caricaturale, dans les statistiques du commerce, puisqu'on a pu compter au cours des dernières années qu'un tiers du chiffre d'affaire du commerce de détail en Russie était réalisé à 
Moscou, chiffre absolument extraordinaire si on prend en compte le fait que la population de la ville ne représente que $8 \%$ de celle de la Fédération. Cela correspond à un pouvoir d'achat théorique moyen par Moscovite quatre à cinq fois supérieur à celui du reste du pays. Il faut néanmoins se méfier de cette statistique, qui est un peu biaisée, car le chiffre en question ne représente pas intégralement des achats effectués par les Moscovites. Il y a des gens qui continuent à faire certains achats à Moscou, notamment sur les nombreux « marchés de gros » et qui ensuite organisent leur petit circuit de distribution, repartant avec un petit camion ou une Jigouli bourrée à craquer de marchandises vers des villes de province.

Le tissu des entreprises a été affecté par des transformations structurelles considérables. L'industrie a connu un déclin spectaculaire. La production industrielle de la ville a diminué de $63 \%$ entre 1990 et 1998, ce qui représente une régression plus importante que ce qui avait été enregistré dans l'ensemble du pays. Au cours des mêmes années, la contraction de la production industrielle pour toute la Russie avait en effet été de l'ordre de $55 \%$. Cette chute vertigineuse de la production a eu des conséquences importante en termes d'emploi. D'après la statistique officielle, le nombre de travailleurs de l'industrie à Moscou a été divisé par deux en très peu d'années. L'essentiel de la contraction de l'emploi industriel se produit entre 1990 ( 1,1 million d'emplois dans l'industrie moscovite) et 1994 (600000 emplois seulement). On peut parler d'un véritable phénomène de désindustrialisation accélérée de la ville.

On observe corrélativement une fulgurante montée de l'économie des services. C'est à Moscou que se crée par exemple, dès le début de la décennie 1990, un marché de la publicité, avec ses agences, ses créatifs (fig. 1). La montée en puissance des services se présente en fait sous deux aspects. C'est d'abord la manifestation d'un mouvement général qu'on a pu décrire dans tout le pays : la montée du petit commerce, une offre de services de proximité (garages, cordonneries, etc.) qui se développe. Ces activités, traditionnellement déficientes en U.R.S.S., sont très visibles dans le paysage urbain, à Moscou comme ailleurs. On a vu se monter dans toutes les villes du pays, petites, moyennes ou grandes, des kiosques (fig. 2), des marchés (fig. 3) et la capitale ne fait nullement exception. Ce qui par contre est plus caractéristique de Moscou, c'est l'apparition d'une économie de services diversifiée dirigée en direction des entreprises. Alors que dans les pays développés ayant une longue tradition d'économie de marché cette palette de services sophistiqués (services bancaires pour les entreprises, bourses, sociétés de conseil stratégique ou financier, etc.) est apparue très progressivement, au cours d'un processus de plusieurs décennies, à Moscou ce tissu s'est constitué à partir de presque rien en dix ans.

Dans un domaine qui m'est proche - le traitement de l'information géographique à des fins économiques - j'ai vu apparaître des sociétés de géomarketing fondées par d'anciens collègues issus de l'Académie des sciences ou de l'Université. Dans le monde des magasins de luxe, tous ceux qui ont pu visiter Moscou dans les cinq ou six dernières années ont été frappés par la présence visible du commerce haut de gamme dans 
l'ensemble du centre de la ville. Cela s'est vu avec les premières rénovations et réappropriations des vieilles implantations commerciales prestigieuses (l'un des tous premiers cas a été dès 1991-1992 le Goum, mais ce n'est pas le seul : un nombre important des anciens "passages » commerçants construits autour de la fin du XIX $\mathrm{X}^{e}$ siècle ont été rénovés pour accueillir des boutiques de prestige). On a aussi réutilisé très vite les implantations commerciales des anciennes rues commerçantes prérévolutionnaires, en supprimant les activités commerciales banales de l'époque soviétique pour y installer des enseignes chic. C'est ainsi que la rue Tverskä̈a (rue Gorki à l'époque soviétique) a en fort peu de temps pris un air de Champs-Élysées.

Dans ce contexte de l'effervescence de l'économie de services, on est frappé par la rapidité avec laquelle s'est constitué un marché de l'immobilier de bureaux. Lors du passage de l'économie administrée au marché, un très grand nombre de nouvelles entreprises ont été créées, qui toutes avaient besoin de locaux adéquats. Au début des années 1990, la ville n'était pas en mesure de faire face à ces besoins. Et en trois ou quatre ans, autour des années 1993 à 1995, la question a été pratiquement résolue; on dispose aujourd'hui de surfaces de bureaux considérables, bien équipées en moyens de communication et en services divers. Certes, les loyers commerciaux sont très élevés, mais on peut considérer que l'offre couvre la demande. Cela se marque de façon variable dans le paysage. Certaines entreprises comme la société pétrolière Lukoil ont réutilisé des locaux qui auparavant appartenaient à des administrations soviétiques. Une autre stratégie, plus spectaculaire, est représentée par la construction ex-nihilo de sièges sociaux : c'est notamment le cas de la Gazprom qui a construit un complexe immobilier dominé par une tour imposante, dans le Sud-Ouest de la ville (fig. 4).

Moscou a réussi son passage. Elle a maintenant le profil d'une métropole mondiale selon les critères internationaux : on y observe une concentration considérable de fonctions de direction d'entreprises, on y a vu apparaître une économie de services diversifiée, capable de répondre aux besoins de ces entreprises, et son profil économique est très international.

\section{Une transformation fermement encadrée.}

L'image de la Russie véhiculée par les médias occidentaux n'est guère attirante. On montre en général la misère, la corruption, la désorganisation et les profits illicites. Il est vrai que la Russie est un pays où il arrive que des hommes d'affaires soient assassinés, où l'économie " grise » a une importance énorme, où déclarer ses impôts selon les formes requises n'est pas le premier souci des entrepreneurs. Tout cela est vrai, mais, à ne montrer que ces aspects-là, on passe à côté de transformations particulièrement intéressantes, notamment à Moscou. Moscou a en quelque sorte endossé le rôle de capitale de l'Antimonde, alors que c'est injustifié. S'il y a de l'ordre quelque part en Russie, c'est bien à Moscou.

Du fait de la création de la Fédération de Russie, Moscou est devenue sujet de la Fédération. Son maire, Youri Loujkov, cumule les pouvoirs de chef de l'administration municipale et de gouverneur. C'est un personnage de premier plan dans la vie politique russe. On sait qu'il a essayé infructueu- 


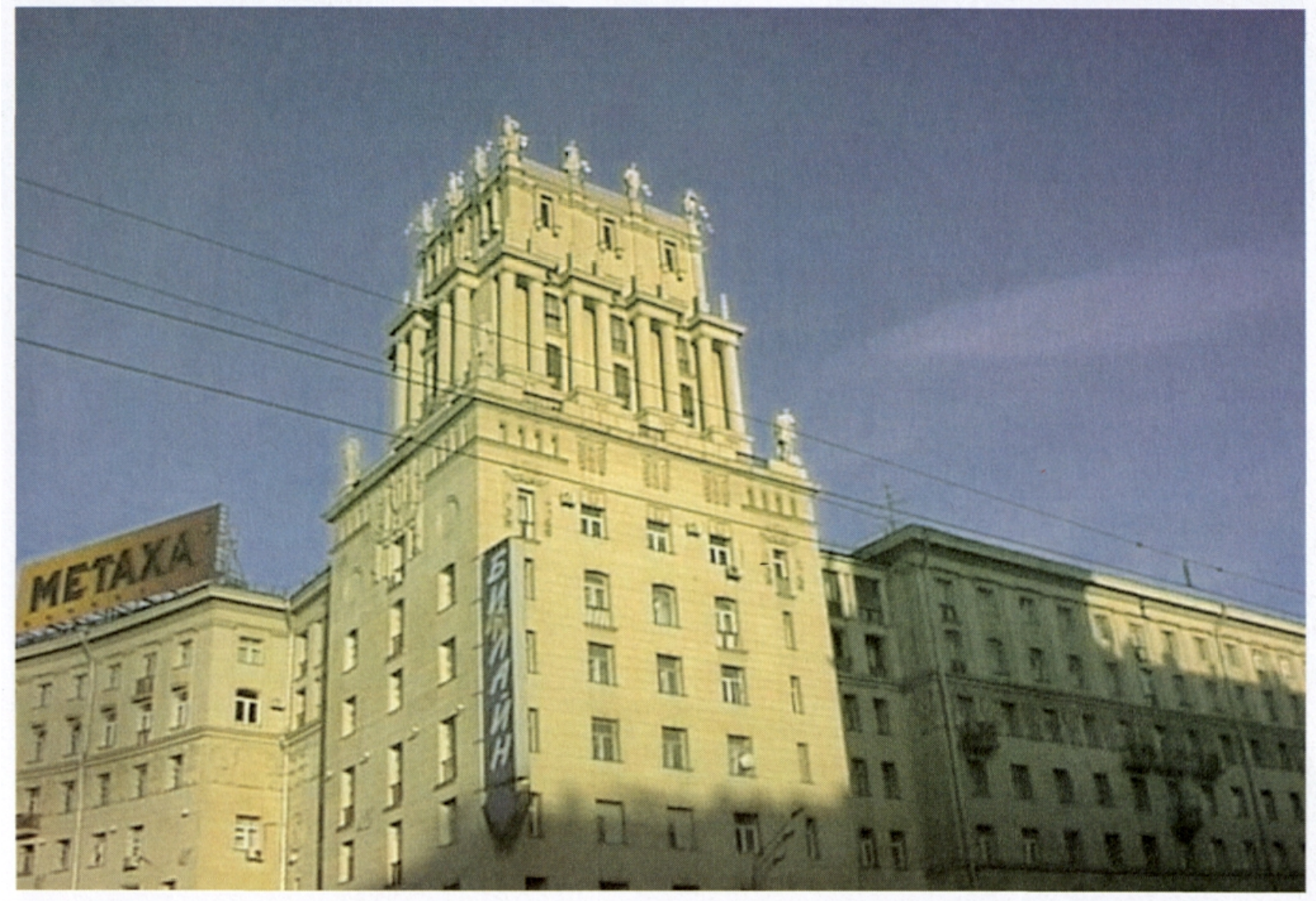

Fig. 1.

Place Gagarine. Immeuble monumental stalinien avec publicités récentes: alcool grec Metaxa, réseau de téléphonie mobile Bee-Line (société russe VimpelCom). 


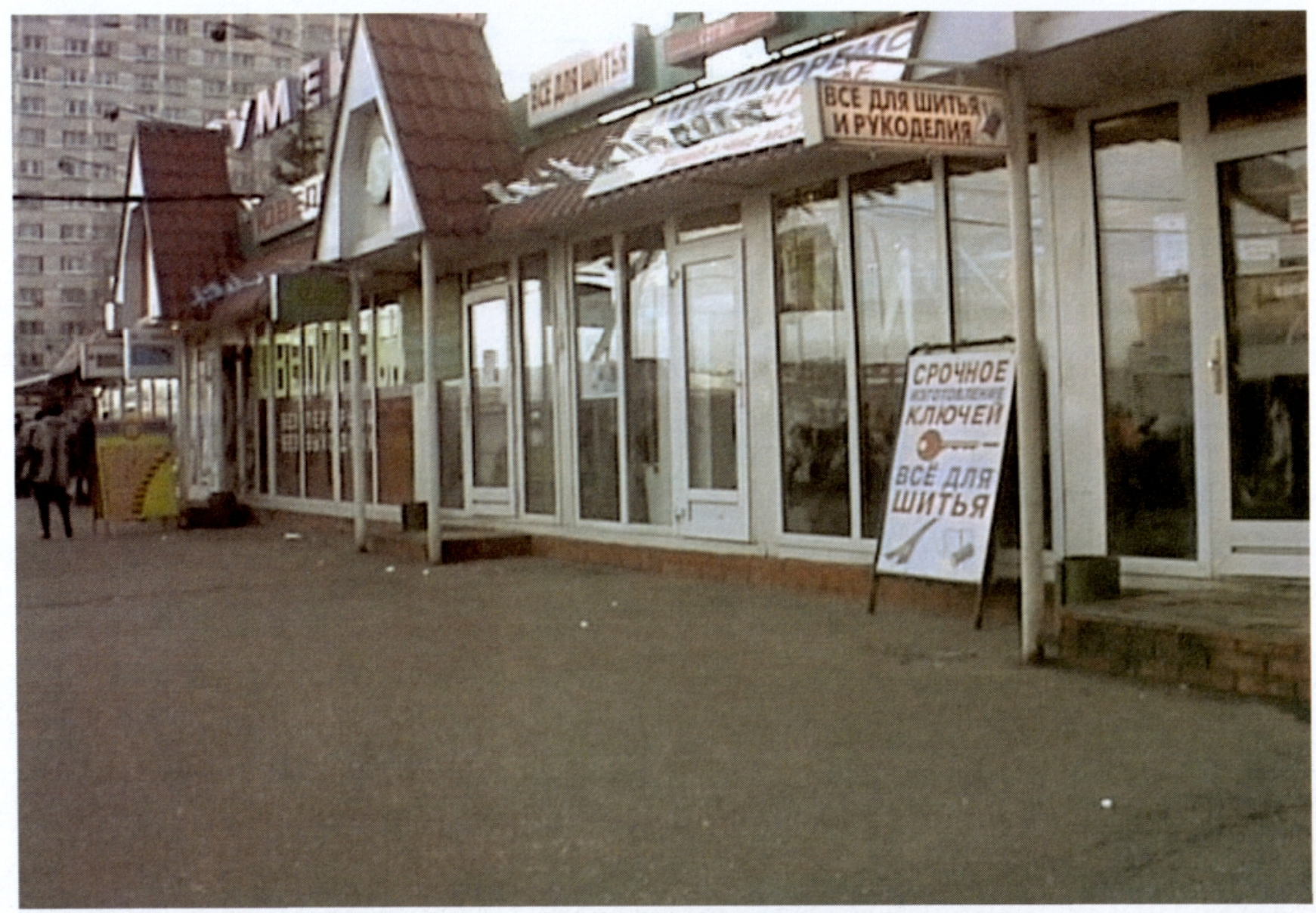

Fig. 2.

Métro Léninski prospekt. Commerces, dont une mercerie et un atelier de reproduction de clés. Bâtiment léger posé sur une dalle de béton. On note l'effort de décoration (auvents en tuiles) qui marque la date récente de construction. 


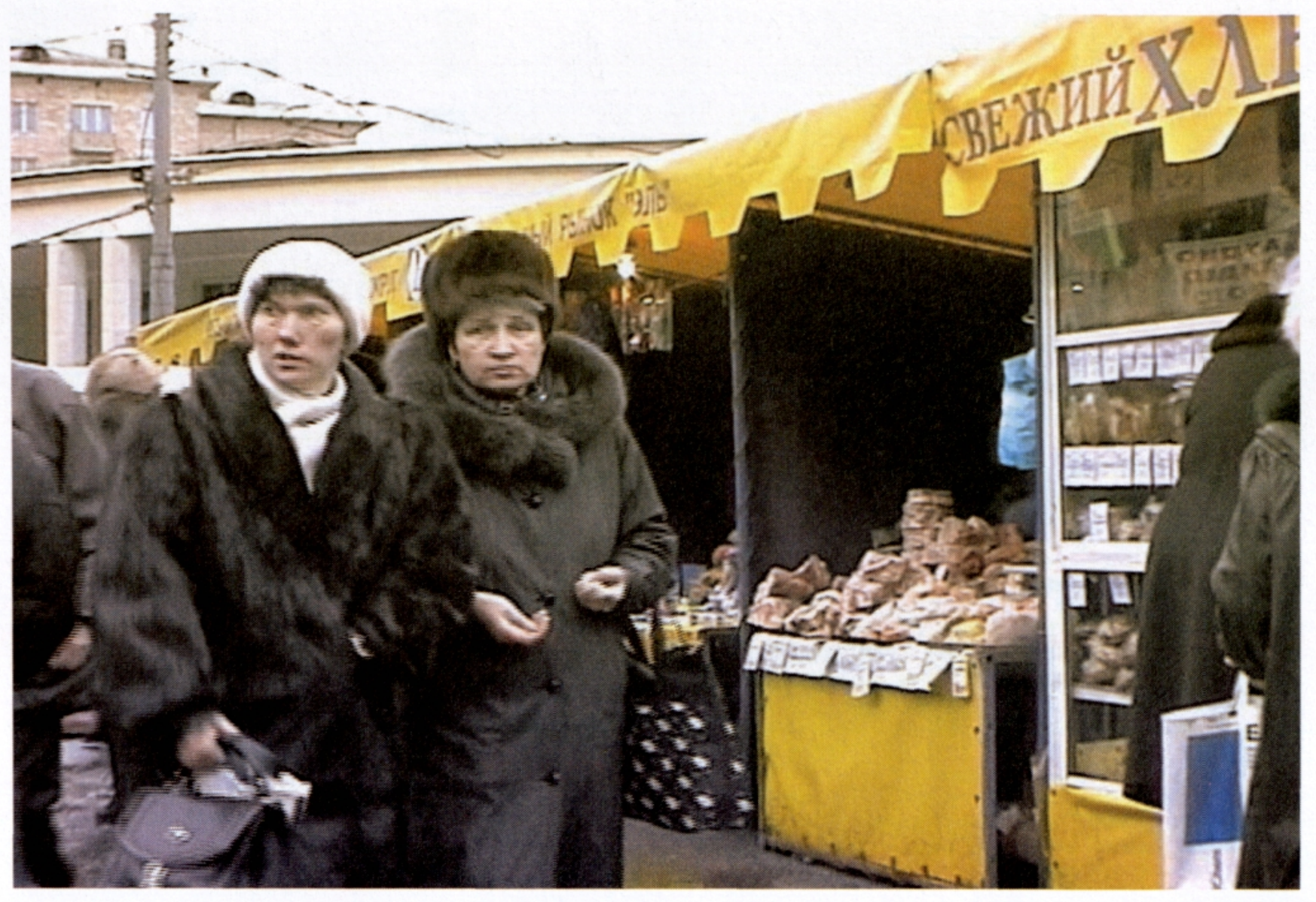

Fig. 3.

Métro Universitet. Stand alimentaire typique d'un marché de sortie du métro. Charcuterie sous emballage plastique, enseigne "Au pain frais ». 


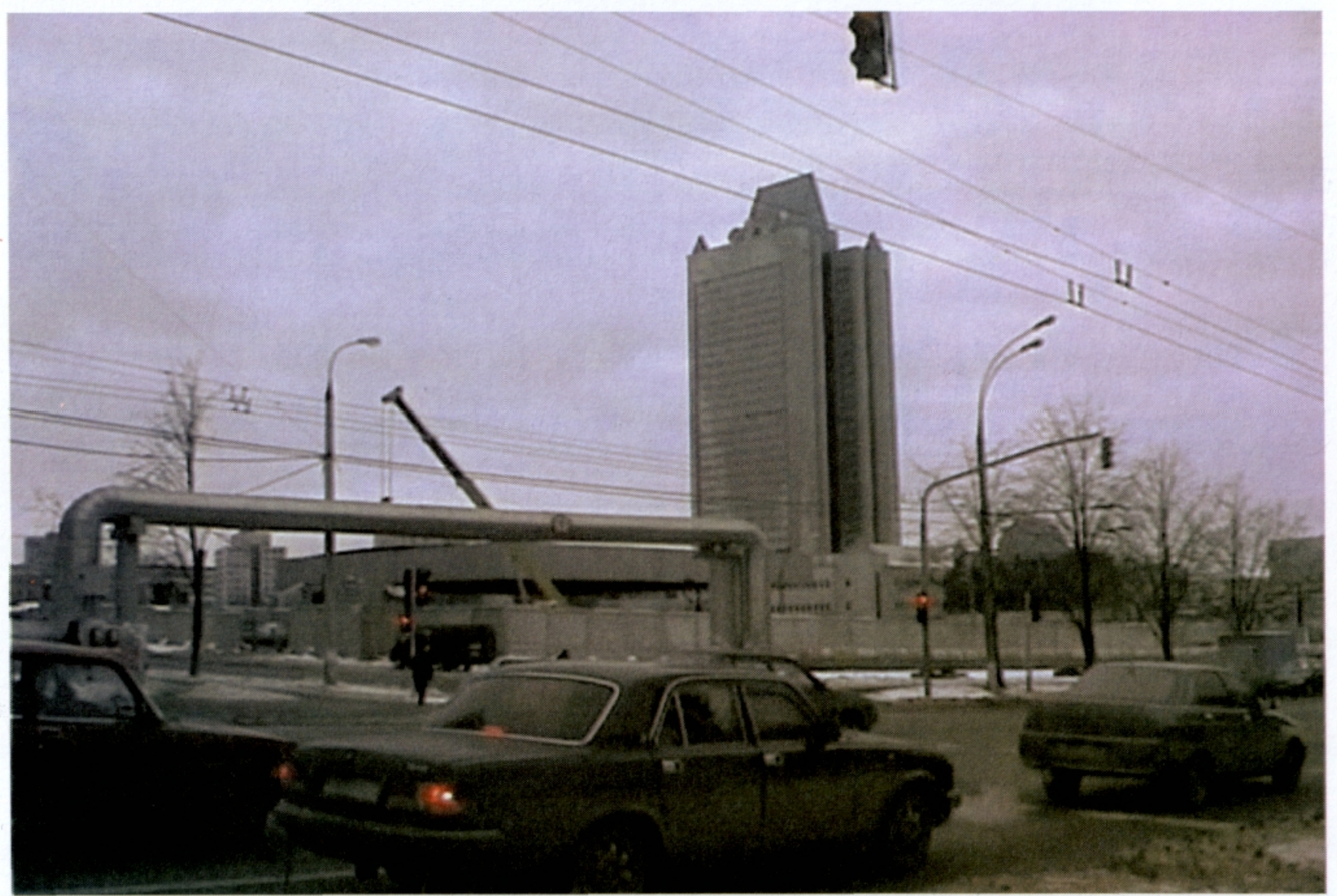

Fig. 4.

Rue Nametkina. Siège social de la société Gazprom, construit au milieu des années 1990. 


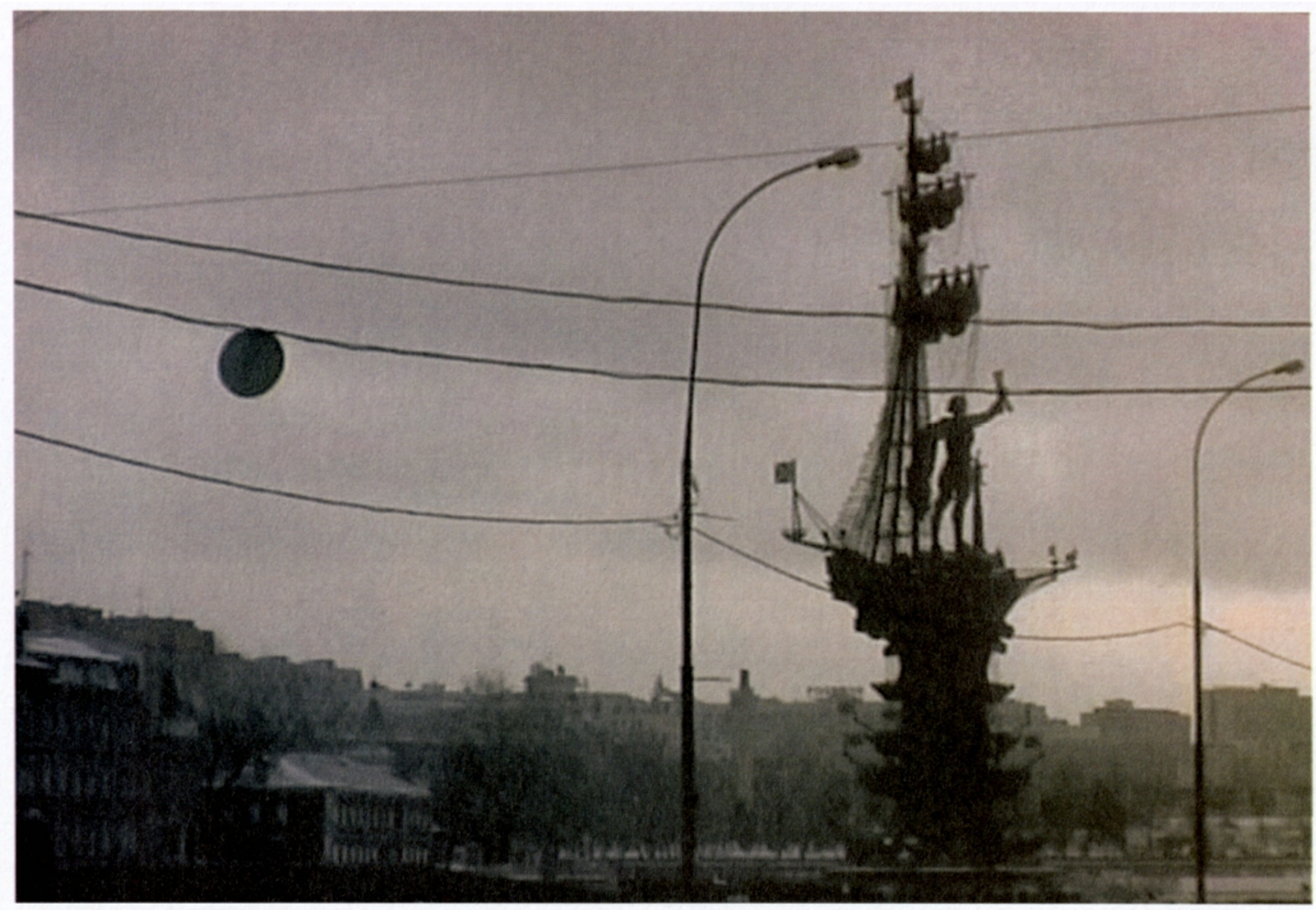

Fig. 5.

Centre ville, sur la Moskova. Monument à Pierre le Grand, milieu des années 1990 (Zourab Tseretelli). La construction de ce monument kitsch gigantesque avait déclenché de violentes polémiques. 


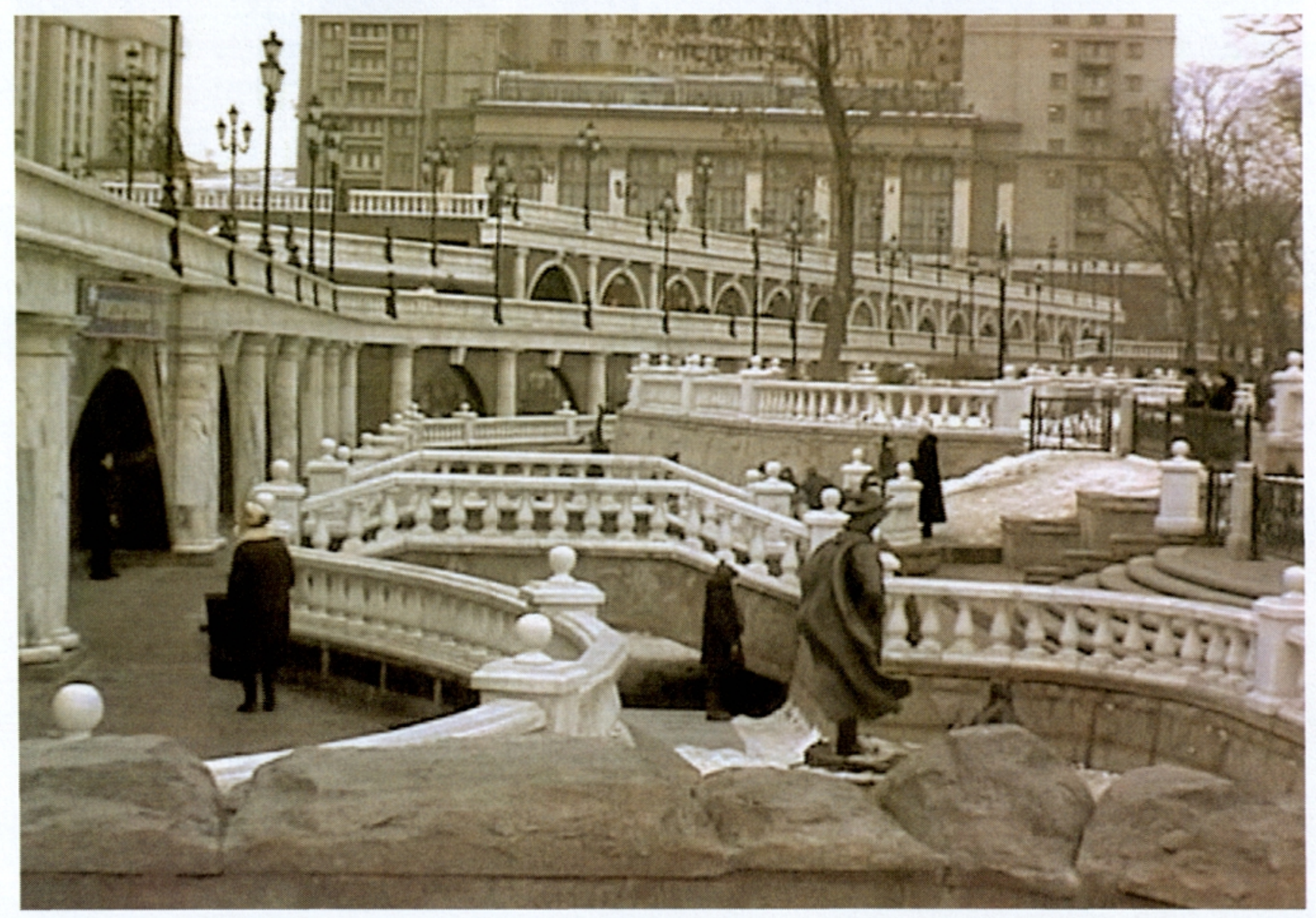

Fig. 6.

Bordure du Jardin d'Alexandre, Place du Manège. Travaux d'aménagement récents (Zourab Tseretelli). Les passerelles enjambent une reconstitution de l'ancienne rivière médiévale, la Néglinka. On remarque la décoration chargée (lampadaires, statues, balustrades). Le centre commercial du Manège est situé derrière les arcades visibles au second plan. Au fond, l'hôtel Moskva. 


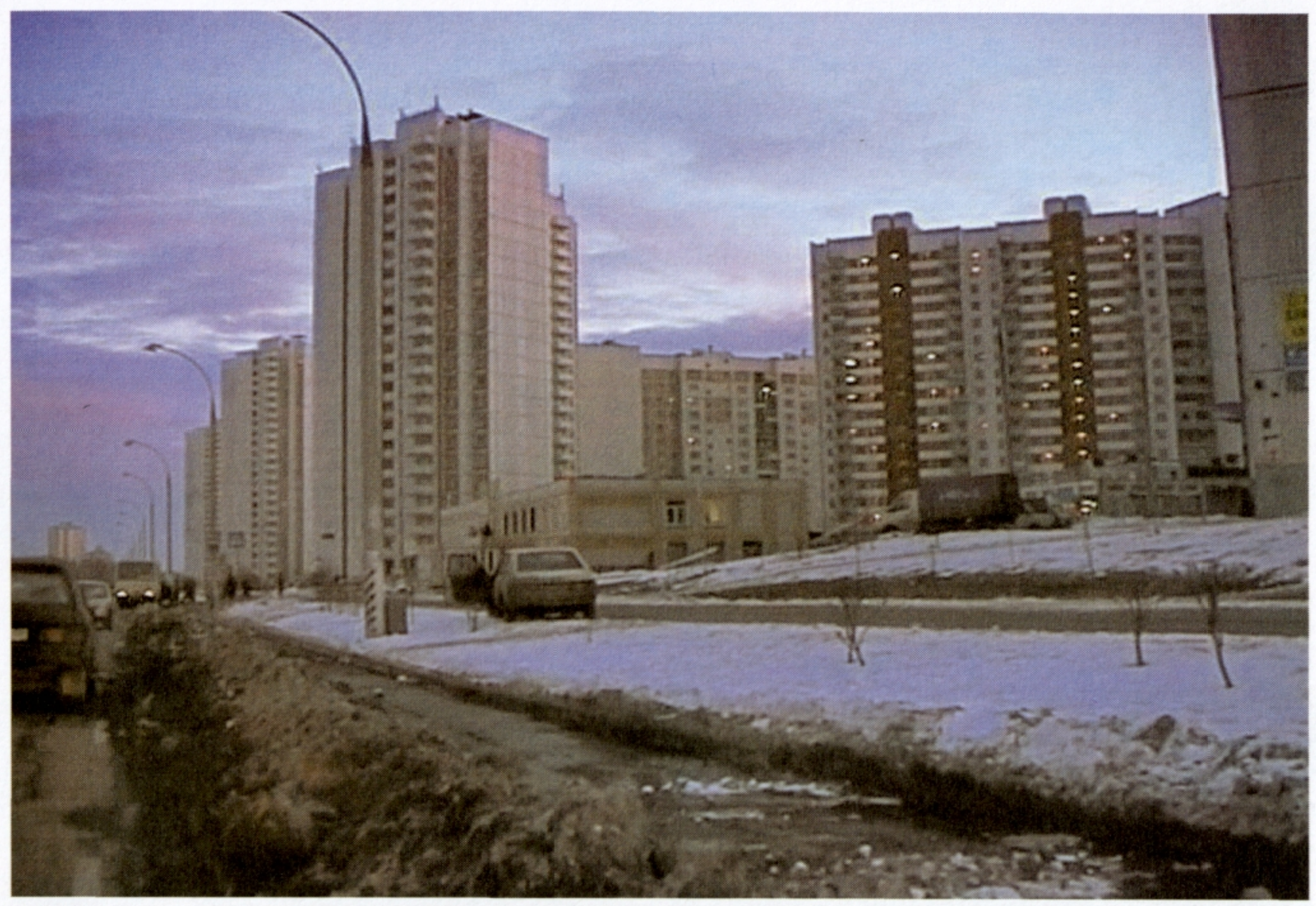

Fig. 7.

Faubourg de Boutovo-Nord, dans le Sud de l'agglomération. Quartier standard d'urbanisation récente: tours et barres de quinze à vingt-cinq étages. Ces immeubles collectifs sont représentatifs des choix architecturaux faits par la mairie pour loger la masse de la population. 


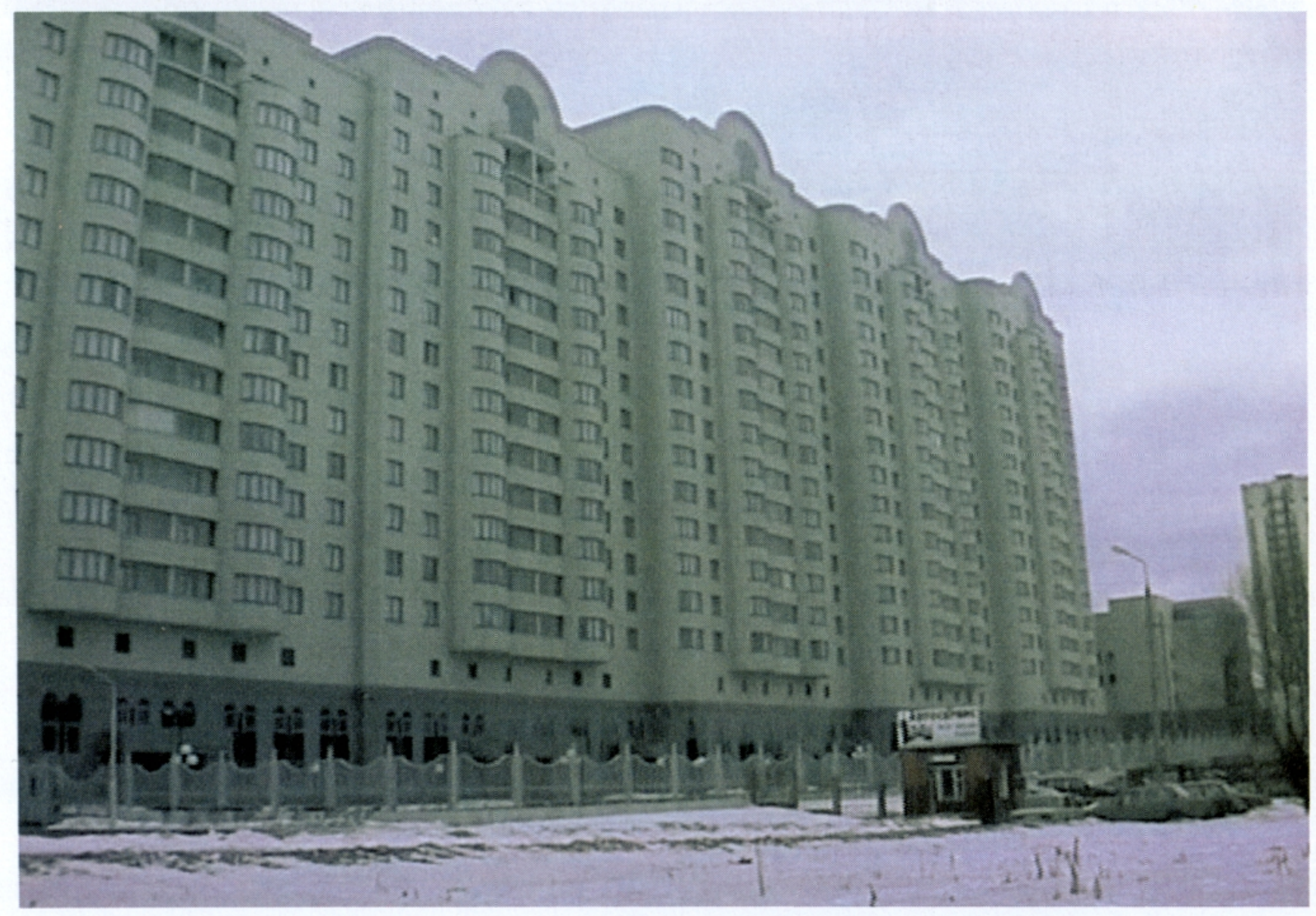

Fig. 8 .

Rue Nametkina. Immeuble de luxe des cadres de l'entreprise Gazprom. La façade est un peu décorée (arcades et balcons). On voit que les immeubles récents situés hors du centre historique sont de grands collectifs, même quand il s'agit de loger des privilégiés. 


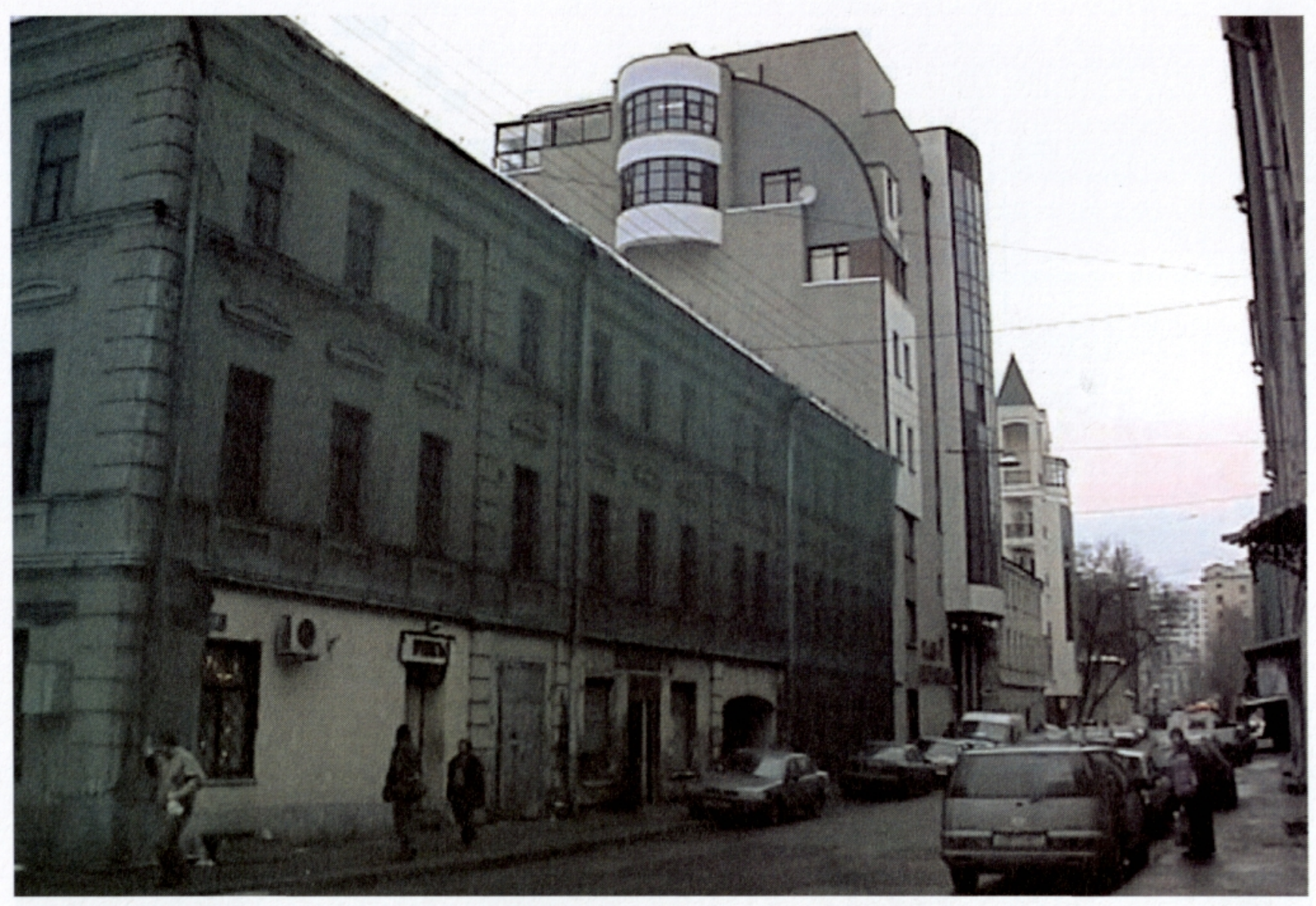

Fig. 9.

Quartier Sretenka (centre historique). Juxtaposition d'un immeuble classique en cours de réhabilitation (sous les filets) et d'un immeuble moderne à l'architecture postmoderne soignée : volumes complexes, tourelles, bow-window. 


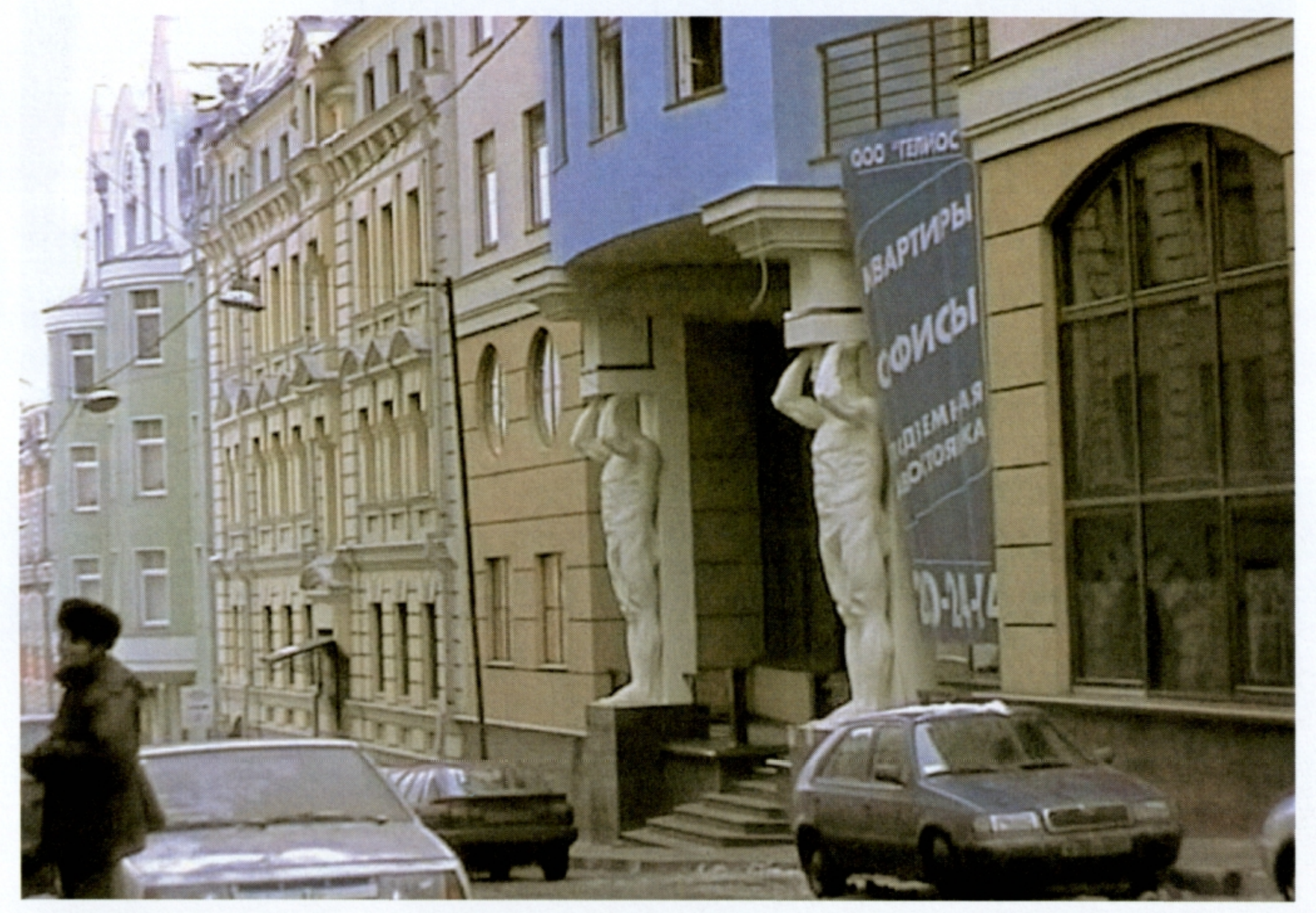

Fig. 10.

Quartier Sretenka, ruelle Posledny. Série d'immeubles rénovés; au premier plan, immeuble mixte de bureaux et d'habitation de luxe. 


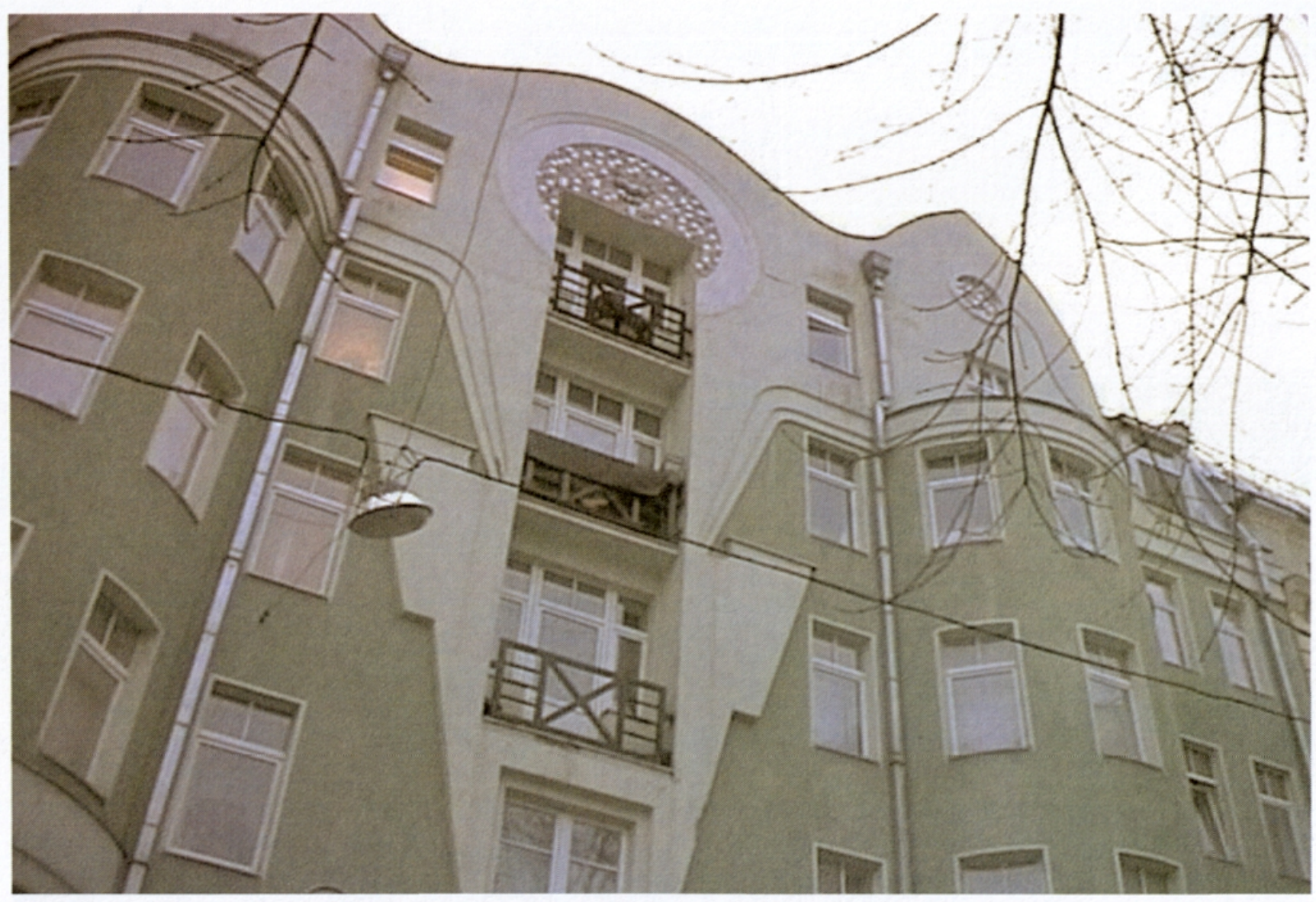

Fig. 11 .

Quartier Sretenka, ruelle Posledny. Immeuble récent imitant le style 1910-1920. 


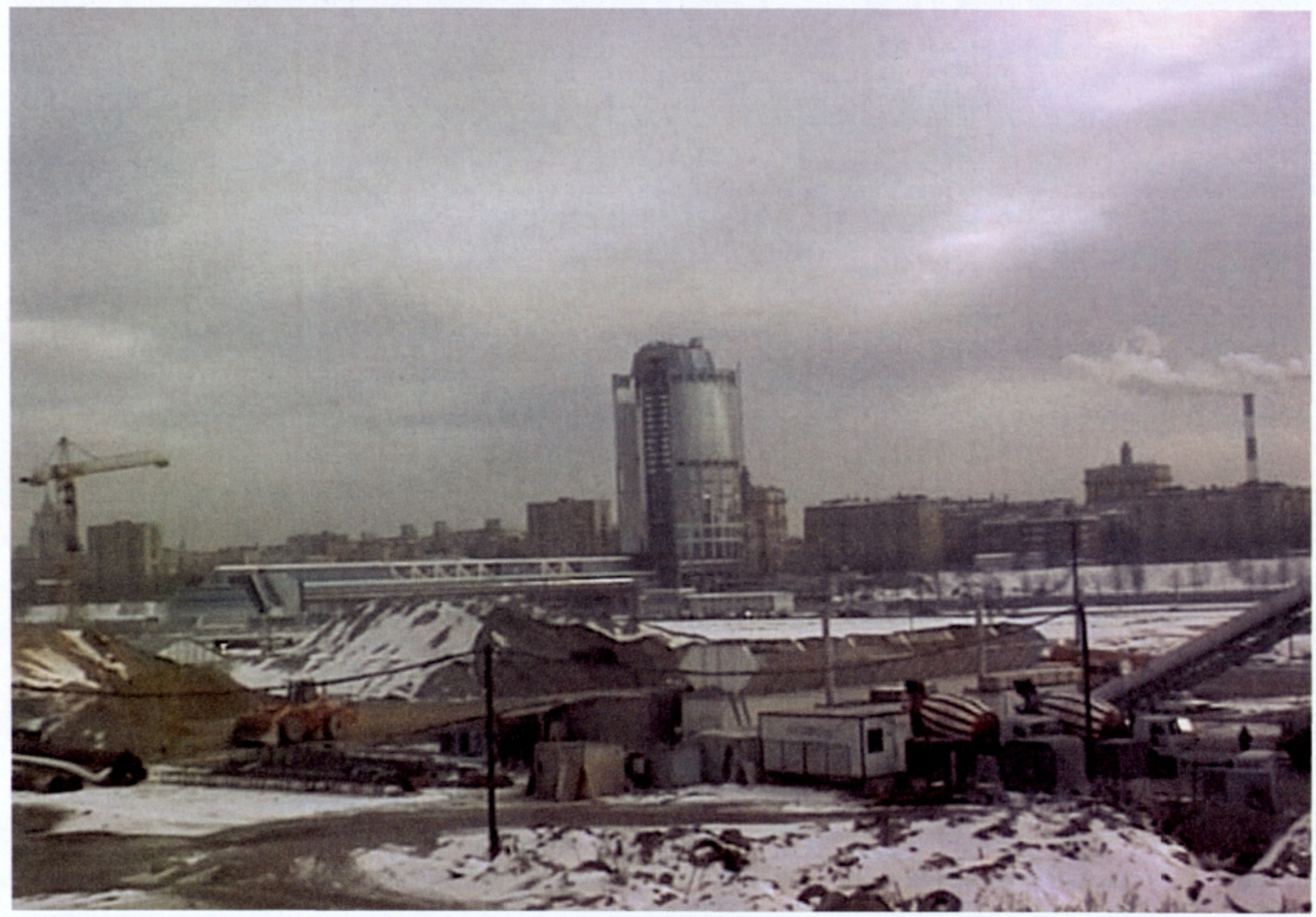

Fig. 12.

Chantier du complexe d'affaires Moscow-City, quartier de Krasnaïa Presnia. La passerelle en verre enjambe la Moskova et conduit, à travers la tour visible au milieu de l'image, à la perspective Koutouzov. 


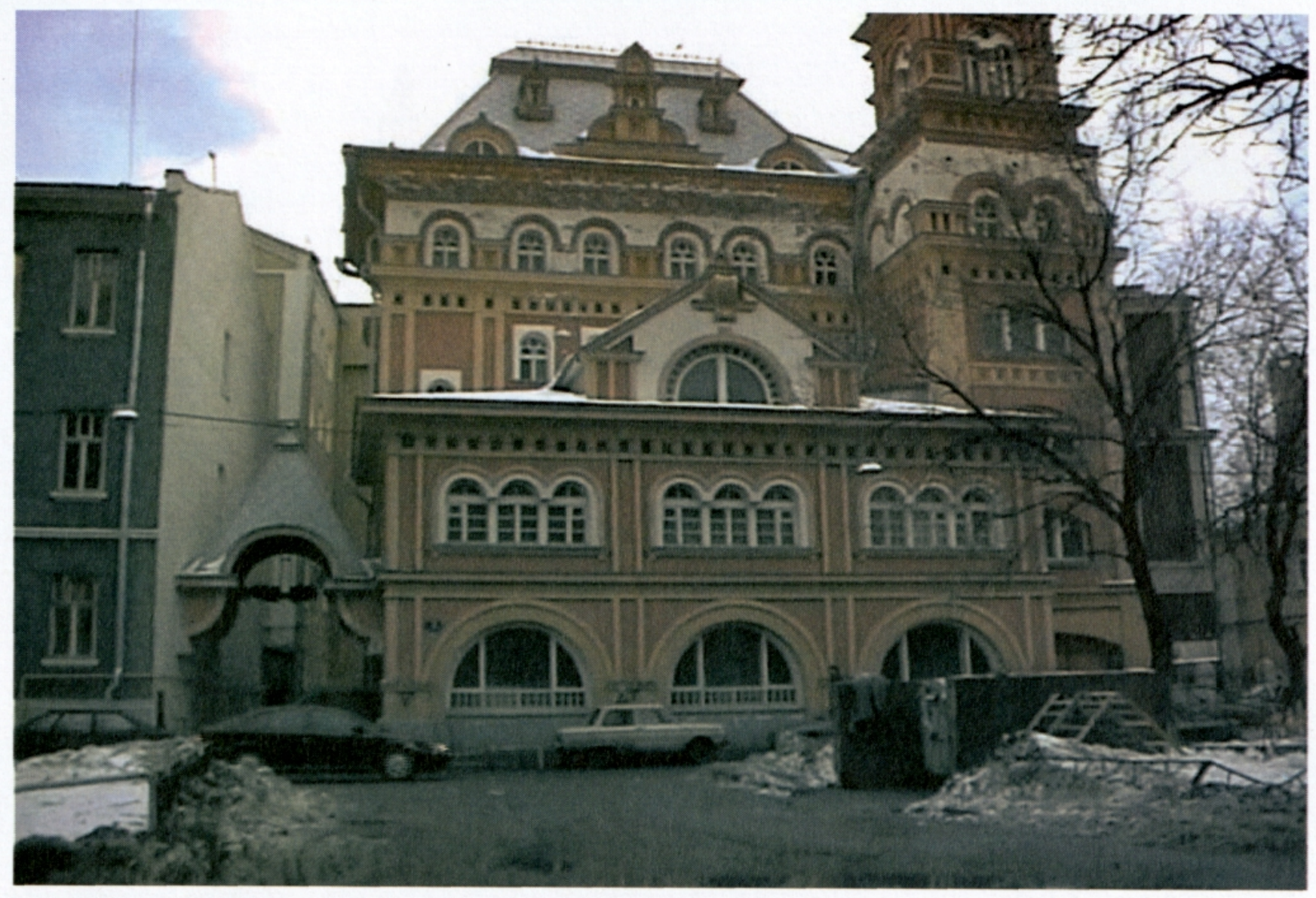

Fig. 13.

Quartier Zamoskvoretchié. Ruelle Khvostov.

Immeuble récent et bigarré pastichant divers styles: évocation d'échoppe de marchand au rez-de-chaussée, citations classiques et Renaissance, imitation du " pseudo-russe » de la fin du XIXe siècle. 


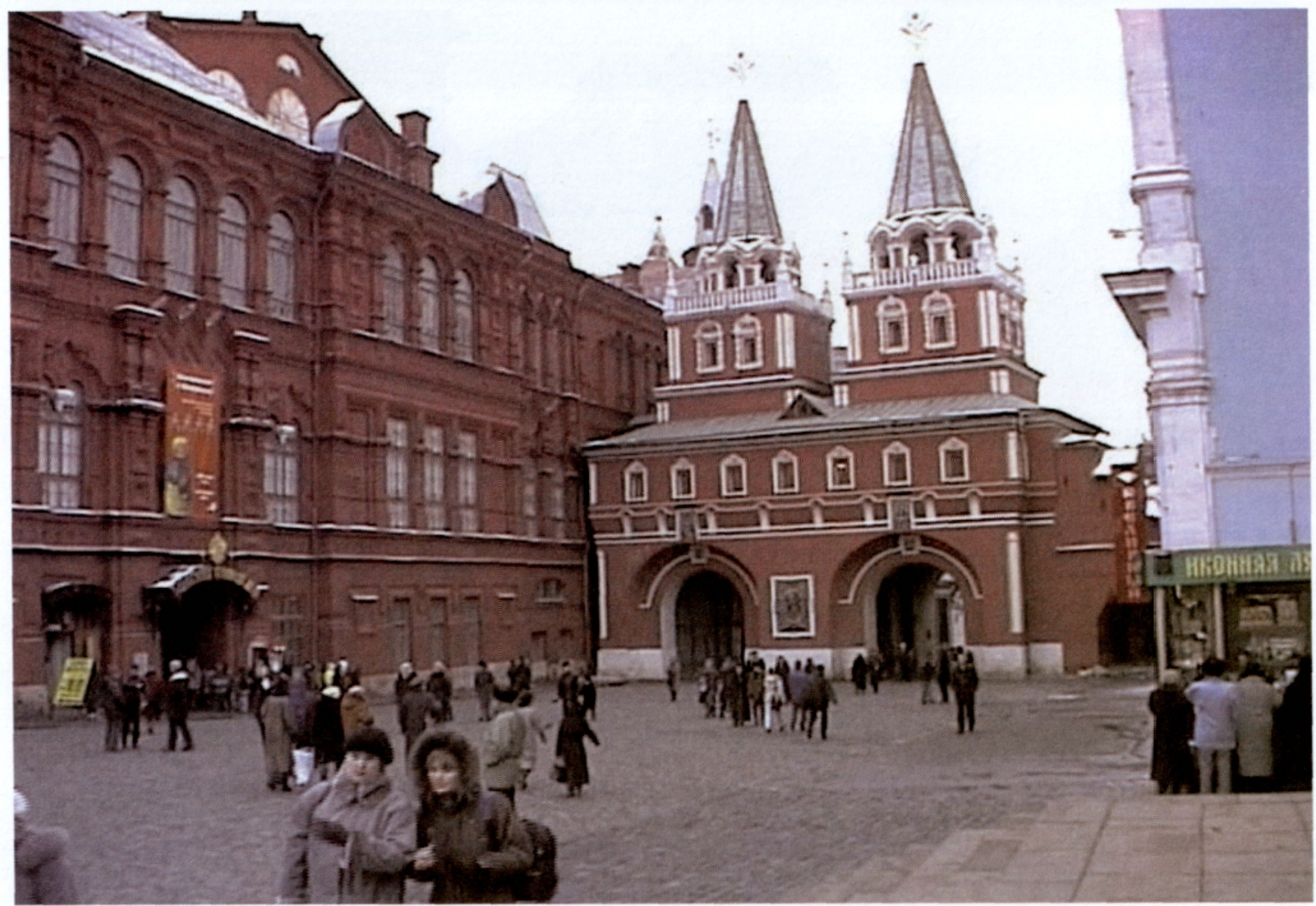

Fig. 14.

Portes de Voskressensk (place Rouge). Ces portes, détruites à l'époque soviétique, ont été reconstruites au milieu des années 1990. 


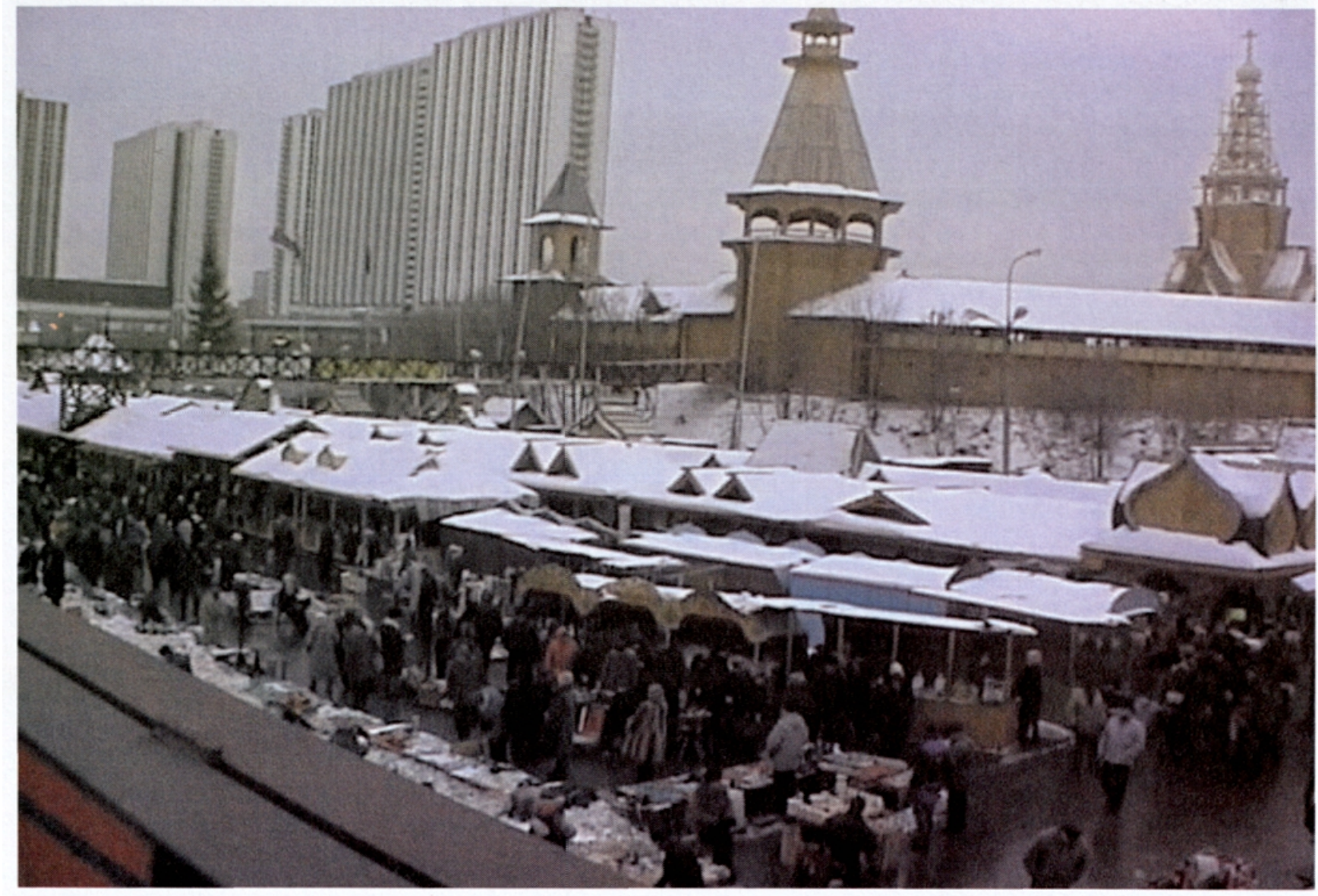

Fig. 15 .

Marché touristique d'Izmaïlovo (Nord-Est de la ville). Faux Kremlin en bois construit en 1998, sur fond du complexe hôtelier construit par les syndicats pour les Jeux olympiques de 1980. 


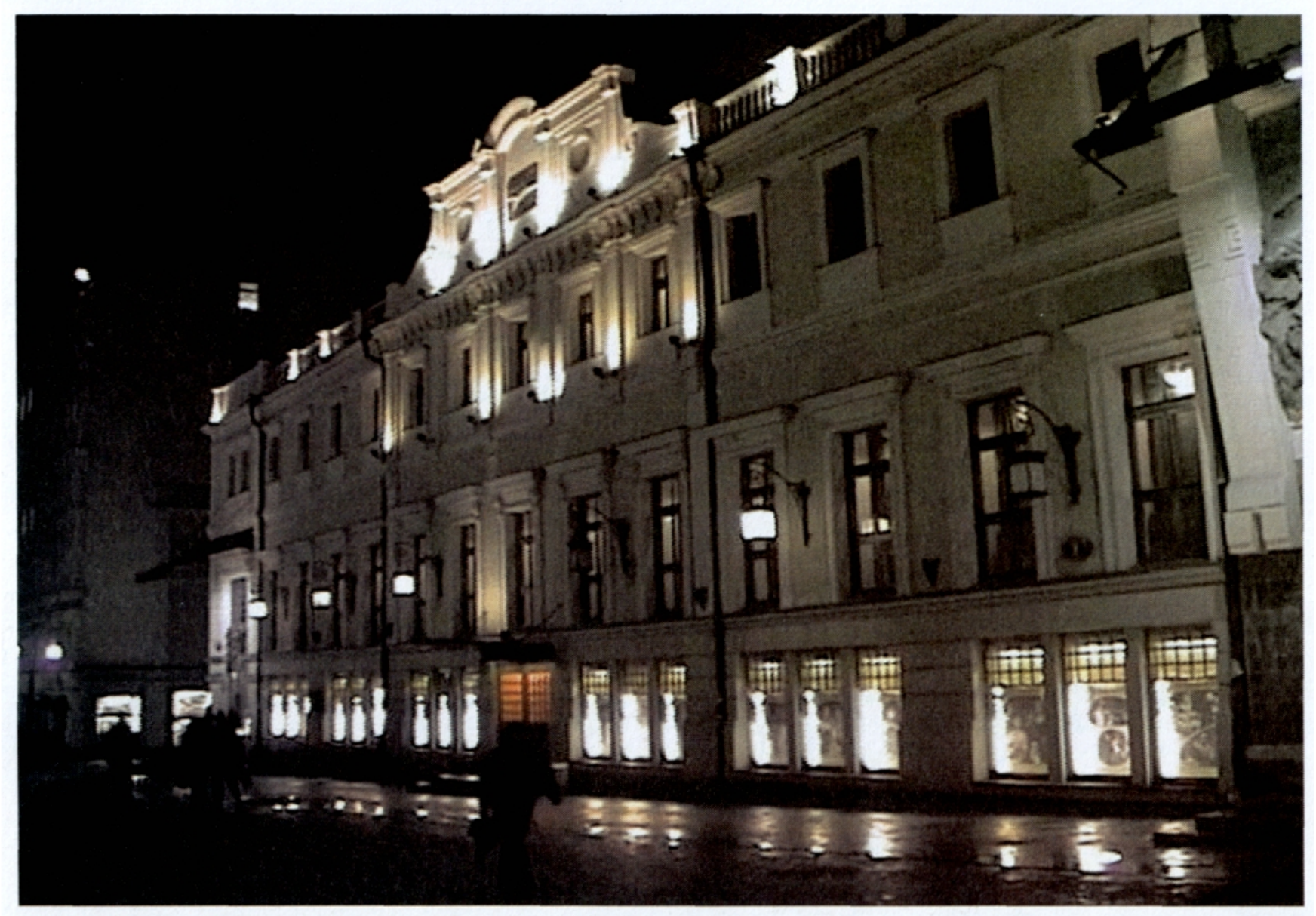

Fig. 16.

Théâtre MKhAT (ruelle Kamergerski, centre historique). Exemple caractéristique de la politique d'éclairage des monuments de la ville, avec mise en valeur de la verticalité. 
sement d'utiliser cette position comme tremplin pour une éventuelle grande carrière politique fédérale en 1998-1999, mais l'habileté remarquable de l'équipe qui a mis en place $\mathrm{V}$. Poutine lui a ôté ses illusions. Il a néanmoins une grande influence.

Il est depuis des années le porteur d'une politique ferme à l'intérieur de la ville. Il s'était opposé durement à l'équipe ultralibérale d'Eltsine au moment de la libéralisation de l'économie, ayant refusé certaines modalités de la privatisation des biens. Il a ainsi fait rentrer beaucoup plus d'argent que prévu dans les caisses de la municipalité, ce qui lui a donné des moyens d'action supplémentaires. Il a refusé de brader le patrimoine, et notamment le patrimoine foncier. L'un des outils les plus importants que Loujkov a mis en place est le système des baux fonciers à 47 ans. La municipalité a le monopole du foncier dans l'ensemble de la ville, elle se contente de concéder des baux, gardant jusqu'ici la propriété du sol. Elle utilise directement ce pouvoir pour structurer, organiser, modeler la transformation urbaine. C'est une politique indéniablement dirigiste. Il ne faut pas voir cela comme l'élément d'un projet réactionnaire, le désir d'un retour à une économie planifiée. Ce dirigisme municipal est au service de l'économie libérale, au service d'un projet de développement des affaires. L'obsession est de transformer la ville en machine à générer de la croissance économique. Les dirigeants de la ville veulent lui assurer un rayonnement véritablement international, en faire une des cités qui comptent dans le monde libéral d'aujourd'hui, ce dont témoigne notamment le lancement du projet de «Moscow City ». C'est une vision de l'économie urbaine et de l'internationalité qui tourne résolument le dos à ce qui a été fait à l'époque soviétique.

La concentration du pouvoir et la centralisation des décisions d'urbanisme expliquent dans une large mesure la rapidité des transformations de la physionomie de la ville. Les projets ont été fermement encadrés par la mairie, en privilégiant la rapidité d'exécution. Les architectes qui avaient un projet de rénovation, de transformation, de réaffectation d'îlots, de rues, d'immeubles, se sont retrouvés face à un pouvoir municipal qui a privilégié les gens qui étaient capables d'aller vite, donc ceux qui étaient en cheville avec les milieux d'affaires de l'immobilier. Ceci a généré et encouragé des formes de collusion et d'affairisme opaque autour de la municipalité. Il est probable que ceux qui dans l'entourage de Loujkov sont en charge des grandes affaires immobilières et des chantiers urbains arrivent à tirer des profits substantiels de cette position. Le personnage le plus flamboyant de cette équipe est sans doute l'artiste architecte Tseretelli, qui a réussi à parsemer la ville d'un certain nombre de ses productions. Le plus évident de ces grands signes est le monument à Pierre le Grand érigé au milieu de la Moskova (fig. 5), mais on pourrait aussi bien citer le travail de rénovation de la place du Manège (fig. 6) ou du zoo.

Néanmoins, la politique municipale est marquée par un souci du maintien du pacte social avec la population. Tout est certes fait pour développer le business dans la ville, mais à la condition que la population moscovite puisse bénéficier d'un certain nombre de retombées. On sait que les services municipaux fonctionnent moins mal qu'ailleurs, que la prise en charge sociale et médicale de la population est meilleure que dans le reste du pays, 
et que l'on continue à faire un effort de rénovation dans le domaine du logement qui est considérable. Le programme le plus populaire d'ailleurs est celui de la suppression des médiocres immeubles à cinq étages de l'époque khrouchtchévienne (пятиэтажки) qui sont en piètre état, et leur remplacement par des logements neufs de qualité correcte (fig 7). Mais on constate que la prééminence accordée aux grands immeubles collectifs n'est nullement remise en cause, renforçant la sévérité du paysage urbain hors du centre ville, et ce, même lorsque l'on construit des résidences de luxe pour la nouvelle bourgeoisie (fig. 8).

Le pacte social a sa traduction dans l'espace urbain. Il y a une évidente segmentation de l'espace à Moscou, acceptée et encouragée par la municipalité. Le centre ville, à l'intérieur du boulevard des Jardins, est laissé au business, aux nouvelles élites, tant pour les activités économiques que pour les lieux d'habitation de prestige. Il n'y a aucun effort de maintien de la mixité sociale dans le Centre. C'est notamment du fait du laisser-faire conscient de la municipalité que les appartements communautaires ont pu si rapidement être rachetés et transformés. On observe ainsi des mutations complètes d'un certain nombre de vieux quartiers, autour de l'Arbat, à Sretenka (fig. 9). En deux ou trois ans, un quartier peut être complètement remis à neuf, et devenir un quartier d'aspect léché, où l'on vend activement des bureaux, où les immeubles de luxe (êlitnye doma) sont gardés par d'impressionnants vigiles et où des caméras vidéos sont accrochées aux façades (fig. 10). Une promenade dans le quartier de Sretenka est à recommander à tout visiteur curieux qui n'est pas allé depuis longtemps à Moscou. Dans ces quartiers anciens rénovés, la profusion de couleurs utilisées pour repeindre les façades donne à la ville un aspect pimpant ou criard, selon les goûts (fig. 11).

L'effort social est fait en périphérie. On y reconstruit des immeubles neufs en remplacement de logements dégradés. De nouveaux quartiers sont également en construction, aux limites de la ville, par exemple dans le quartier de Boutovo dans le Sud.

\section{L'image changeante d'une ville : la russification de Moscou.}

Dans les vingt/trente dernières années de la Moscou soviétique, la transformation urbaine était impulsée par la volonté de donner le visage d'une ville internationale, d'une cité préfigurant le futur communiste. Moscou devait devenir le symbole de l'internationalisme et d'une modernité que les Moscovites ont pu juger très agressive à certains moments. La construction du siège du Comecon, des immeubles du quartier du Nouvel Arbat (sur l'avenue dénommée à l'époque Kalinine) témoignaient d'une volonté de faire de Moscou le prototype de la cité socialiste, avec très peu de marqueurs spécifiques russes, sauf une église conservée de-ci de-là. Aujourd'hui, on est dans le cadre d'un projet national, même si économiquement la ville s'est ouverte au monde capitaliste. Il s'agit d'ancrer Moscou dans le passé russe. La seule exception notable - et récente - dans le centre est la construction du monumental quartier d'affaires Moscow-City au bord de la Moskova, qui doit signer l'appartenance de la ville au cercle des villes du «big business » (fig. 12). Mais, pour le reste, il s'agit de refaire de Moscou le Cœur de la 
Russie. La politique architecturale dans le centre est entièrement dirigée vers la production de références historiques (fig. 13). Il y a de ce point de vue une réelle convergence entre les autorités fédérales, la municipalité et même la hiérarchie religieuse. Le slogan «Moscou est le cœur de la Russie » (Moskva, serdce Rossii) a été repris dans des publicités - affiches, spots télévisés - diffusées par l'Église orthodoxe pour appuyer la campagne de collecte de fonds en vue de la reconstruction de la cathédrale du SaintSauveur. Ce ré-ancrage de Moscou dans le passé russe va passer par un mouvement non pas de réparation ou de restauration, mais bien de recréation du passé. Les éléments les plus évidents, les plus spectaculaires de cette recréation sont certes la construction de la cathédrale du Saint-Sauveur mais aussi les grandes transformations de la Place Rouge : on a reconstruit l'église de Notre-Dame de Kazan et les Portes de Voskressensk (fig. 14). C'est un vrai travail de refabrication du passé. Le discours implicite est le suivant : « nous allons vous prouver que Moscou est une ville profondément russe : nous la parsèmerons pour cela de symboles russes ». Il ne s'agit pas d'une restauration dans le sens du respect de l'authenticité historique. On prend les symboles comme ils viennent. L'intervention de la religion dans ce dispositif est significative. Comme l'un des éléments forts de ce mouvement de recréation du passé est la réimplantation d'églises, on en construit beaucoup, en général là où une église ancienne avait été détruite, mais dans un style de pastiche. Domine l'évocation de divers styles russes traditionnels. On donne aussi dans l'imitation du néo-russe ou néo-byzantin de la fin $\mathrm{du} \mathrm{XIX}^{\mathrm{c}}$ siècle. Je ne pense pas que les autorités, qui favorisent ce mouvement de reconstruction d'édifices religieux, veuillent réellement redonner à la religion dans la cité la place qu'elle pouvait occuper avant la Révolution. S'il s'était uniquement agi de recréer des lieux de culte en nombre important, on aurait pu lancer des concours architecturaux et voir émerger des édifices pourquoi pas aussi originaux que la cathédrale d'Évry de Mario Botta. Les autorités municipales auraient pu encourager çà et là quelque expérimentation architecturale. Mais cela n'a pas été le cas. Le projet est à l'évidence de remettre du symbole religieux traditionnel là où il y en avait. La religion est instrumentalisée. Cette recolonisation de Moscou par les églises n'est certes pas le symbole d'un retour massif de la population moscovite à la pratique religieuse.

Un autre aspect de la recréation du passé est la rénovation de façades classiques dans le centre. Très souvent des immeubles très dégradés du XIX ${ }^{\mathrm{c}}$ siècle font l'objet de chantiers de rénovation, ce qui signifie qu'on les abat presque entièrement pour reconstituer après reconstruction une façade classique. Ces rénovations violentes du patrimoine architectural sont certes encouragées par la fragilité des immeubles de construction ancienne (en brique, bois et façade stuquée). Un grand nombre de sièges d'entreprises ont l'apparence de palais classiques, mais si l'on entre, on est dans un monde de béton et de verre. Il est donc plus intéressant d'interpréter ces mouvements non en termes de restauration mais de recréation, de pastiche, d'intégration de symboles du passé dans la physionomie d'une ville modernisée. C'est vrai qu'elle est du coup devenue très colorée, il y a un aspect agréable dans la façon dont elle a été totalement rénovée. 
Un autre aspect de ce mouvement est l'évocation de la Russie profonde, de la vieille Russie. On a vu apparaître dans un certain nombre de lieux les jardins qui bordent la cathédrale du Saint-Sauveur par exemple - des chapelles de bois, des architectures en rondins. Sur l'anneau des Boulevards, l'un des espaces publics du centre dont le mobilier urbain est le plus soigné, il y a des jeux pour enfants entièrement en bois, avec des figures qui évoquent les petits jouets traditionnels. C'est une évocation de la russité rurale. L'endroit le plus caractéristique est le marché touristique d'Izmaïlovo, dont l'enceinte a été reconstruite en forme de kremlin en bois en 1998. Cette évocation surréaliste du passé russe est visible sur le fond du complexe hôtelier construit pour les Jeux olympiques de 1980 (fig. 15). Ces postiches de bois transforment Izmaïlovo en Disneyland, en temple du kitsch russe.

Enfin, il faut mentionner la politique d'éclairage nocturne du centre ville et des grands axes : l'objectif de ce travail est de mettre en scène, de valoriser au mieux les éléments les plus spectaculaires du patrimoine urbain : encore un travail destiné à imposer l'image d'une ville grandiose, splendide, qui sait mettre en valeur son passé (fig. 16).

(Ce texte est le résumé de la conférence prononcée dans le cadre du colloque de Bordeaux.)

\section{Quelques références :}

Eckert D. (2001) «Moscou, capitale russe, métropole mondiale ? », in Portraits de grandes villes : Société, pouvoirs, territoire, Jalabert G. (coord.), Presses Universitaires du Mirail, coll. Villes et Territoires.

Eckert D., Kolossov V. (1999), La Russie, Paris, Flammarion, coll. Dominos, 128 p.

Radvanyi J. (2000), La Nouvelle Russie, Paris, Armand Colin, 418 p.

Vendina O. (1997), "Le nouveau visage de Moscou et la contribution de la municipalité à la modernisation de la ville», Actes du Colloque «Les problèmes culturels des grandes villes », Paris.

http://www.cybergeo.presse.fr/culture/vendina/vendina.htm 